\title{
Criminologie
}

\section{Femmes et justice : problèmes de l'intervention}

\section{Marie-Andrée Bertrand}

Volume 16, numéro 2, 1983

Les femmes et la justice pénale

URI : https://id.erudit.org/iderudit/017182ar

DOI : https://doi.org/10.7202/017182ar

Aller au sommaire du numéro

Éditeur(s)

Les Presses de l'Université de Montréal

ISSN

0316-0041 (imprimé)

1492-1367 (numérique)

Découvrir la revue

Citer cet article

Bertrand, M.-A. (1983). Femmes et justice : problèmes de l'intervention.

Criminologie, 16(2), 77-88. https://doi.org/10.7202/017182ar d'utilisation que vous pouvez consulter en ligne.

https://apropos.erudit.org/fr/usagers/politique-dutilisation/ 
FEMMES ET JUSTICE:

PROBLÈMES DE L'INTERVENTION

Marie-Andrée Bertrand*

Quel que soit le sexe de la personne qui souffre l'intervention pénale, celle-ci pose des problèmes fondamentaux de plusieurs ordres. Problèmes de droit, de légitimité, d'équité, d'utilité; problèmes ontologiques, problèmes économiques.

Il apparaît utile de rappeler ces limites du droit pénal dans le cadre d'un article portant sur les femmes et la justice. En effet, le droit à intervenir de façon contraignante est une bien grave affaire. On ne saurait «disposer qu'en tremblant de la vie et des biens des hommes» (Beccaria, 1764, 1965, p. 2) et les réflexions que nous inspireront l'examen et la critique du droit à intervenir auprès des femmes à partir de leurs infractions et à la lumière de leur condition socio-historique enrichiront un débat déjà animé, débat millénaire, sur les fondements du droit pénal et sur le droit de contraindre.

\section{DES PROBLÈMES «MORAUX» DU DROIT PÉNAL}

Le premier et, sans doute, le plus grand problème qui se pose à la criminologie et à l'intervenant "pénal" relève de ce que les marxistes appellent l'ontologie, les chrétiens, la morale et à peu près tout le monde le bien et le mal. Quelle est donc la nature de l'acte criminel? Existe-t-il vraiment au-delà des déterminations socio-culturelles fort relatives qui supportent des consensus et des valeurs, des actes «mauvais en soin?

À cette question, métaphysiciens et moralistes ont souvent apporté des réponses tellement claires, tellement définitives qu'on se prenait à douter que là se trouvât la réalité... Nous n'entreprendrons pas de rappeler, dans le cadre de cet article, toute la force (écrasante) avec laquelle tant de philosophes, Platon lui-même dans les Lois, ont tenté de nous convaincre qu'il existe un «bien» et un «mal» en soi.

Au fut et à mesure où s'accroissent nos connaissances et notre compréhension des mécanismes par lesquels le pouvoir se crée, s'exerce et étend son domaine, au fut et à mesure où l'analyse historique et l'analyse transculturelle nous montrent la fragilité, voire l'inexistence de prohibitions éternelles et universelles, nous sommes

* Professeur à l'École de criminologie, Université de Montréal. 
définitivement ébranlés dans nos certitudes de «bons» et de «bienpensants"):

Le jugement «bon» n'émane nullement de ceux à qui on a prodigué la «bonté»! Ce sont bien plutôt les «bons» eux-mêmes, c'est-à-dire les hommes de distinction, les puissants, ceux qui sont supérieurs par leur situation et leur élévation d'âme qui se sont eux-même considérés «bons", qui ont jugé leurs actions «bonnes», c'est-à-dire de premier ordre, établissant cette taxation par opposition à tout ce qui était bas, mesquin, vulgaire et populacier. C'est du haut de ce sentiment de la distance qu'ils se sont arrogés le droit de créer des valeurs et de les déterminer : que leur importait l'utilité! (F. Nietzsche, La généalogie de la morale, 1885 (1964), p. 28).

\section{LES PROBLĖMES DE LÉGITIMITÉ DU DROIT PÉNAL}

\subsection{NOS INTERVENTIONS SONT-ELLES FONDÉES EN DROIT?}

L'intervention pénale, faut-il le rappeler, devrait, en principe, être fondée en droit. Par intervention pénale, nous entendons, en effet, celle qui impose par la contrainte une peine, de quelque nature que ce soit : restriction de la mobilité, des fréquentations, modification du style de vie, des habitudes de boire, sanctions financières, contribution obligée aux services socio-sanitaires, sentences communautaires qui peuvent constituer des peines semi-privatives de liberté et, finalement, bien sûr, toutes les sentences privatives, c'est-à-dire la prison avec ou sans congés.

Or, si nos interventions pénales sont généralement fondées juridiquement, elles ne le sont pas toutes et pas toutes également. Certaines de nos interventions auprès des mineurs, des membres de certains groupes ethniques, des citoyens marginaux, voire même des femmes, ne répondent pas à cette exigence première d'un fondement en droit. Toutes bienveillantes qu'elles se prétendent, elles ne sont pas non plus fondées sur un droit qui reconnaît l'égalité de tous devant la loi. En effet, c'est bien connu, plusieurs provisions des codes pénaux sont discriminatoires, selon le sexe, selon l'âge, voire selon la race.

\subsection{UN DROIT PÉNAL LÉGITIME}

Le droit pénal, s'il prétend entraîner des interventions légitimes, doit lui-même être caractérisé par la légitimité. Or, tel n'est pas toujours le cas, loin de là. Certains parlent d'inflation pénale (Landreville et al., 1980). Je parlerai de l'illégitimité du droit pénal.

Pour parler d'illégitimité, il faut d'abord parler de légitimité. 
En principe, dans nos sociétés démocratiques, seuls les consensus clairs, portant sur des valeurs chères, méritent d'être protégés au prix de sanctions pénales. Comme le rappelle la Commission (canadienne) de Réforme du droit (spécialement dans les Confins du droit pénal et dans Notre droit pénal, 1975, 1976), tel n'est pas le cas de la majorité des 50000 infractions prévues aux lois pénales canadiennes, soit dans le code criminel, soit dans les lois fédérales, les lois provinciales ou les lois municipales. Le Canada d'ailleurs n'est pas le seul pays à souffrir de cette obésité pénale. Les parlements modernes ont beaucoup abusé de leur pouvoir de créer des infractions. On cherche en vain à propos de $90 \%$ d'entre elles les consensus sociaux, «les états forts de la conscience collective» (Durkheim) au nom desquels s'est créée l'incrimination.

Dans le cas de certaines infractions, on peut parler de leur désuétude : les consensus ont probablement déjà existé, mais se sont effrités avec le temps ou bien font l'objet de telles remises en question qu'on ne saurait parler vraiment de consensus. Tel est le cas, selon la Commission de réforme du droit, d'infractions bien controversées comme l'avortement, les actions indécentes, la bigamie, la polygamie, le complot, les infractions relatives aux drogues, l'inceste, l'obscénité, la pornographie, le jeu, la désobéissance aux lois et ordonnances du tribunal (Commission de réforme du droit du Canada, 1976, p. 35). Mais dans d'autres cas, les consensus n'ont probablement jamais existé. Par exemple, on se demande ce qui a bien pu fonder les infractions qui consistent à «inscrire des paris», "affecter des pratiques de magie» ou à "posséder un véhicule à moteur muni d'un appareil produisant un écran de fumée». Les locomotives de cette époque devaient sûrement être hors la loi.

Non seulement la criminalisation doit-elle répondre à des critères précis dont les quatre principaux sont les suivants :

$1^{\circ}$ que l'action cause un préjudice grave à autrui;

$2^{\circ}$ qu'elle viole des valeurs fondamentales à un point tel qu'elle cause un tort réel à la société;

$3^{\circ}$ que l'application du droit pénal n'entraîne pas des torts ou des violations aux valeurs fondamentales;

$4^{\circ}$ qu'on ait des raisons de croire que l'incrimination peut apporter une solution aux problèmes soulevés par le crime.

Mais la prohibition règlementaire (par exemple, nos lois provinciales sur l'alcool ou sur la circulation routière) doit elle-même être contenue dans des limites raisonnables et jugées socialement 
pertinentes, moralement «justifiées «(on doit être assuré, là aussi, que la règlementation ne cause pas plus de tort que de bien, qu'elle ne lèse pas les droits et les valeurs fondamentales, qu'elle est utile, etc.)

Ce sont là, essentiellement, les critères exigés par la majorité des théoriciens du droit pénal pour fonder l'incrimination. Citons, entre autres F.A. Allen, C. Beccaria, J. Bentham, P. Devlin, L.L. Fuller, H.L.A. Hart, J.S. Mill, H.L. Packer, E. Schur, M. Miaille, etc.

Enfin, le droit relatif aux infractions touchant la propriété devrait être simplifié et reconsidéré à la lumière d'une nouvelle évaluation du rôle de la propriété dans la société (CRD, 1976, op. cit., p. 36).

\subsection{L'ABSENCE DE FONDEMENT Ȧ L'INCRIMINATION}

DE LA MAJORITÉ DES INFRACTIONS COMMISES PAR LES FEMMES

Examinons maintenant la criminalité officielle des femmes à la lumière de cette première réflexion. Nous parlerons d'accusations plutôt que de condamnations, les statistiques touchant ces dernières marquant un retard considérable, au Canada.

C'est un fait universel: de $40 \%$ à $90 \%$ de toutes les femmes accusées, selon les pays, le sont pour des crimes contre la propriété, sans violence. Au Canada comme ailleurs, il s'agit de vols à l'étalage d'objets de peu de valeur, de faux chèques et de chèques sans provisions. Ce groupe d'accusées est suivi, au Canada, par un autre : la cohorte des femmes arrêtées pour avoir enfreint les lois sur l'alcool (environ $25 \%$ des accusées). Vient ensuite le groupe des accusées de simple possession de cannabis (de 6 à $7 \%$ des accusées), les auteures d'infractions aux lois sur le cautionnement, les femmes qui ont «troublé la paix», celles qui sont accusées de prostitution (toujours bien plus nombreuses que les hommes), de méfaits publics, de voies de fait et, finalement, les femmes qui sont accusées de s'être «introduites par effraction". Si l'on excepte les deux derniers chefs d'accusation: les voies de fait et le crime qui consiste à s'introduire par effraction (qui représentent chacun $2 \%$ du total des accusations portées contre les femmes au Canada), il tombe sous le sens que les infractions dont les femmes sont coutumières n'ont rien de criminel ${ }^{1}$.

1. Le nombre des femmes accusées de vols avec violence a crû au Canada depuis quelques années. Mais ces accusées ne représentent que la moitié de $1 \%$ du total des femmes inculpées. 
La définition criminelle de ces infractions est remise en question ou par l'absence de consensus social à propos des valeurs outragées ou, plus simplement, par l'absence de tort réel à autrui ou à la collectivité. Touchant les infractions contre la propriété, celles dont les femmes sont le plus souvent accusées ne peuvent ni ne doivent être traitées devant une cour de justice criminelle. Il faut réserver le procès pénal aux crimes véritables (CRD, 1976, p. 36).

Est-ce à dire qu'aucune contrainte n'est justifiée à l'endroit de $90 \%$ ou même $95 \%$ des accusées? - Il y a contrainte et contrainte. Le droit de contraindre à dédommager, à restituer, à contribuer à restaurer le bien-être social, personnel, physique que le délinquant a menacé ou détruit par son action novice semble tout à fait fondé, à propos du vol, de la fraude, des méfaits publics, des lésions corporelles, des blessures, etc., dédommagement, réparation, restauration par des mesures civiles, voire administratives. Contrainte physique? Peine privative? - Sûrement pas. Ce n'est ni létigime, ni justifié, ni utile.

Touchant les «vrais» crimes dont les femmes sont accusées : vol qualifié $(0,5 \%$ des accusées), introduction par effraction ( $2 \%$ des accusées), voies de fait graves ( $2 \%$ des accusées), homicide volontaire et involontaire $(0,08 \%$ des accusées $)$ - le droit d'intervention peut s'étendre à la contrainte physique, si l'accusée constitue un danger réel, mais ne doit pas s'étendre à la contrainte physique s'il s'agit d'actes tout à fait situationnels dont la répétition apparaît improbable. C'est le cas de la majorité des actes de violence s'étant produits à l'intérieur de relations domestiques, affectives, en autant bien sûr que le facteur déclenchant soit neutralisé.

\section{LE CARACTĖRE DÉMOCRATIQUE DE LA CRÉATION DES LOIS}

Au-delà des problèmes de légitimité et des problèmes de "moralité» qui ne cessent d'entourer toute la question de l'intervention pénale, d'autres questions encore, presque aussi fondamentales, jaillissent de l'écart entre le modèle créé par le législateur et le fondement «moral» ou «ontologique» de ce modèle. Les mécanismes sociaux et psychologiques de la création de la loi, le processus de sélection des valeurs, les valeurs qu'on protège par opposition à celles qu'on décide de ne pas protéger, la hiérarchie des valeurs au vu de l'importance sociale ou du degré de danger social comme élément de la criminalisation, tout cela pose les grandes questions à la criminologie, les questions «radicales», c'est-à-dire celles qui sont à la source 
même de la pratique de la criminologie contemporaine et de celle de tous les temps.

La création de nouveaux délits ${ }^{2}$ se fait sous la pression de groupes d'influence dont l'appui électoral et financier apparaît non négligeable aux hommes qui détiennent le pouvoir. Ces groupes d'influence : membres d'un parti politique, chefs religieux, services de police, groupes de parents, syndicats, etc., s'efforcent, plus ou moins habilement, directement ou indirectement, de faire passer dans les lois leur conception d'un ordre moral et surtout de ce qui menace cet ordre. On n'a qu'à penser au puissant Lobby qu'entretiennent, dans plusieurs pays, les corps de police, le moment venu d'abolir la peine capitale, voire de décriminaliser la simple possession de drogues, cette infraction mineure et dénuée de fondement légitime apparaissant aux policiers comme nécessaire à la répression du trafic et de l'importation, en tout cas ce sont les rationalisations qu'ils nous servent. Il n'est pas réaliste, même si on peut se prendre à rêver qu'ils le fassent, d'exiger de ces groupes d'influence et d'intérêt que leurs revendications embrassent tout le «bien commun». Il est normal que les groupes d'influence aient des intérêts particuliers. Ce qui est moins désirable et surtout moins légitime, c'est que les institutions parlementaires d'un pays démocratique endossent des vues partisanes et soient la proie de groupes minoritaires d'opinion ou de pressions opérées par de puissantes industries.

S'il paraît inutile, voire nocif d'inventer et de maintenir l'incrimination quand la légitimité disparaît, il n'est pas moins illégitime d'intervenir ou moins nocif de le faire quand le modèle créé par le législateur s'éloigne trop du consensus social et moral.

\section{L'INTERVENTION PÉNALE AUPRĖS DES FEMMES : UNE SORTE DE CRIME?}

Ce préambule n'est certes pas inutile pour discuter de l'intervention pénale auprès des femmes. La majorité des embuches que nous réserve cette intervention se trouve déjà épelée dans les paragraphes qui précèdent. En effet, c'est probablement à propos des infractions commises par les femmes que la légitimité, le fondement même des lois pénales sont le plus souvent et le plus radicalement remis en question. Il se trouve que les délits qu'on leur reproche comptent bien souvent parmi ceux qui reposent sur le moindre consensus

2. Et la conservation de ces vieilles matières sans fondements qu'on n'arrive pas à abroger. 
social, si jamais il y a eu consensus sur la prostitution, la simple consommation de cannabis, le fait de troubler la paix, etc., et ce sont là les délits dont «l'immoralité» est la moins démontrable. Touchant les infractions mineures contre la propriété, nous avons rappelé (supra) comment des organismes aussi pondérés que la Commission de réforme du droit juge nécessaire de repenser tout ce chapitre du code pénal. On pense aussi à l'avortement, à l'homicide involontaire commis à l'intérieur d'un couple amoureux, etc.

Les dangers sociaux que les femmes font courir à la collectivité ou à quelques-uns de ses membres par leurs actions criminelles sont si marginaux, si peu nombreux, que le fait de les incriminer semble relever de la fabrication mâle. Fabrication du processus de sélection des valeurs par des législateurs masculins, valeurs protégées hier encore comme la stabilité du noyau familial, la natalité, la fidélité des épouses (sinon des époux), la chasteté des femmes.

Qui niera que dans la dialectique qui préside à la création du droit pénal, lequel fonde le droit à intervenir, des rapports de dominant/dominé se sont depuis toujours exprimés entre des hommes d'une part et des femmes d'autre part, des rapports de dépendance de la part des femmes, dépendance juridique, économique, affective avec tout le cortège des servilités, des infériorités, de l'absence d'autonomie morale, sociale, qu'entraînent ces rapports d'inégalité?

C'est dans ce contexte et sur cette toile de fond que je voudrais parler du caractère criminel de certaines interventions auprès des femmes.

Toute action professionnelle qui puise sa justification dans des lois qui ont perdu leur légitimité parce que les consensus sociaux ne les supportent plus, toute intervention qui reprend à son compte une définition du «bien» comme répondant à un ordre social défini par un groupe en vue d'assurer ou d'augmenter son pouvoir, sa richesse, son savoir; toute intervention qui s'inspire aussi d'une sélection des valeurs en faveur d'un groupe dominant sur un groupe dominé m'apparaît comme inique. J'ai tenu le même langage pour décrire les manipulations grâce auxquelles les adultes imposent leurs valeurs privilégiées à des mineurs, sans se soucier de la légitimité de ces valeurs, de leur actualité, non plus que des écarts entre le fondement du droit pénal et les modèles que les législateurs nous donnent (Bertrand, 1977).

Nous l'avons vu : des problèmes généraux, de caractère moral et tenant à la légitimité, se posent à propos du droit pénal et des inter- 
ventions qu'il commande à l'endroit de tout citoyen. Des questions encore plus aiguës et plus nombreuses sont soulevées par les chapitres du droit pénal qui servent, très particulièrement, à incriminer les femmes. Les fondements de ces infractions sont encore plus fragiles que ne le sont ceux qui servent à l'incrimination des actions reprochées traditionnellement aux hommes et aux jeunes gens.

Mais nos problèmes ne font que commencer, car une fois ces doutes semés dans nos esprits touchant la valeur de nos interventions ou même le droit à intervenir dans la vie des femmes délinquantes, se pose un autre problème énorme qui est celui du mode.

Quel sera donc le mode le moins inique, le plus approprié, si toutefois l'intervention est légitime, si elle est utile, si elle s'impose?

Tout au long de cet article, j'ai évitél'expression «intervention socio-pénale" pour ne parler que de l'intervention pénale. À mon avis, il faut sortir de l'hypocrisie que génère un certain paternalisme moral et social qui nous fait justifier nos interventions au nom d'un «bien-être», «développement», «mieux être», «maturation», dont nous croyons que nous pouvons gratifier les victimes de nos interventions. Il s'agit ici d'interventions pénales, comme je l'ai dit plus haut, de celles qui contraignent et qui imposent une peine. Comment intervenir auprès des femmes, au nom de lois pénales visant à protéger un ordre social conçu par des hommes pour des hommes, sinon pour confirmer les femmes dans leurs rôles, dans leur situation de dépendance, dans leur instrumentalité archaïque? Que tenteronsnous de "restaurer» par cette intervention, sinon le statut traditionnel que sanctifient les règles du droit pénal?

Je terminerai en relevant deux des plus terribles obstacles à une intervention qui prétend restaurer une certaine équité dans les statuts réciproques et une certaine autonomie chez les victimes de l'intervention. Ces deux obstacles sont : le sexe des législateurs et (très souvent) des intervenants, et le caractère non désiré par le sujet de l'intervention.

Sur cette hégémonie morale mâle que signifie bien le code criminel et d'ailleurs toutes les lois pénales, tout n'a pas été dit, loin de là. Le caractère discriminatoire, sexuellement discriminatoire, des lois pénales n'est pas complètement disparu. Là même où l'on trouve peu de discrimination dans la lettre de la loi, spécifiant le sexe de l'agresseur ou de la victime, ou prévoyant des peines différentes selon le sexe, perdure encore, sauf exception, un modèle de société, des prohibitions qui sont l'envers des valeurs. Personne ne niera que le droit pénal n'évolue pas très vite. 
Touchant le caractère contraignant de la peine et l'aspect imposé de l'intervention procédant du droit pénal, qu'on me permette de renvoyer le lecteur à l'un de mes articles: «Réflexions critiques sur l'intervention et les limites du droit à intervenir" (Santé mentale au Québec, 6, 2, novembre 1981).

$J$ 'y rappelle les distinctions qui s'imposent entre des interventions appelées explicitement par le sujet ou sollicitées par lui; des interventions appelées implicitement et des interventions imposées. Le fait que l'intervention est «appelée» explicitement ou implicitement, confère une certaine légitimité à l'intervenant. Mais combien puissantes doivent être nos raisons d'intervenir, surtout de façon intrusive, lorsque nous ne sommes pas sollicités de le faire par les intéressés. Dans le domaine pénal, ce cas est la règle. Nos int rusions, car les méthodes douces ne sont pas courantes en criminologie, devraient être freinées par les considérations qui précèdent touchant leur faible légitimité ou leur illégitimité, mais devraient être aussi fortement influencées, par le souci de ne pas répéter le rapport dominant/dominé, homme/femme, puissant/faible, pour ne pas parler des rapports des classes sociales, qui caractérise la relation intervenant/criminel, ou intervenant/délinquant, dans le domaine pénal. L'intervention qui aboutit à accroître des rapports de dépendance, de soumission, d'inégalité entre les hommes et les femmes, entre les législateurs et les délinquants, entre les marginalisants et les marginalisés, cette intervention ne peut être que criminelle.

En effet, même le plus cynique des moralistes l'admet, il y a un crime en soi, c'est la volonté efficace de perpétuer les inégalités, d'accroître les dépendances, de ne pas profiter des contacts obligés des femmes avec le système de «justice» (!) pénale pour rompre le cercle de leur dépendance.

Autrement, il faudra bien accepter de parler du système d'injustice pénale.

Finalement, la règle qui devrait présider à nos interventions auprès des délinquantes s'applique tout autant, sinon davantage, quand il s'agit des victimes sauf qu'à l'occasion celles-ci seront des clientes volontaires du système de justice pénale. Le "crime», là aussi, consisterait à nous introduire dans leur vie pour d'autres motifs que celui de les rendre capables de faire face à leur agresseur sans notre aide.

\section{LES FEMMES COMME AGENTS D'INCRIMINATION}

Récemment, des groupes de femmes ont contribué de façon importante et souvent spectaculaire à faire émerger une volonté 
publique de sanction pénale sévère à l'endroit de quelques infracteurs.

On peut dire sans exagérer que certains groupes féministes attisent la vindicte populaire à l'endroit des violeurs et des promoteurs de pornographie.

Les femmes, des femmes, mènent donc une action criminalisante.

Souvent elles-mêmes déresponsabilisées ou irresponsabilisées par rapport à leurs propres crimes (on veut les voir complices même lorsqu'elles sont auteures principales), et d'ailleurs plus souvent qu'à leur tour victimes des crimes des hommes, voilà que les femmes adoptent, collectivement, des attitudes rétributives, punitives, vengeresses. Triste retour des choses!

Que penser de cela?

Pour les tenants de l'intervention pénale minimale, pour ceux qui considèrent que l'incrimination est un mal et que seule la certitude d'un tort réel et sérieux à autrui et à la collectivité, la justifie, certaines des revendications des féministes sont bien gênantes.

Tel est le cas, par exemple, de l'action menée par certains groupes de femmes contre la pornographie. On ne voit pas bien en vertu de quelle logique on peut être à la fois pour la décriminalisation de la prostitution et pour la criminalisation de la pornographie. S'il est souhaitable que les femmes et les hommes se voient reconnaître le droit d'exercer le métier de «prostitué» (j'emploie le mot malgré sa connotation méprisante, faute d'un meilleur terme) et que c'est là une activité qu'il ne faut pas pénaliser tant que personne n'est exploité : ni le client ni le ou la prostitué, — on ne comprend pas bien pourquoi un éditeur et un cinéaste qui auraient choisi de faire porter leurs livres et leurs films sur des matières érotiques devraient être punis par les lois pénales, tant que leurs clients sont "volontaires» et que le libraire, l'éditeur, le cinéaste et le propriétaire de cinéma ne procèdent pas à de la promotion agressive et insistante.

À propos du viol, tout semble bien compliqué et émotivement chargé, mais tentons quand même d'aborder cette infraction dans le contexte d'évolution culturelle et sexuelle qui a marqué les 20 dernières années. Il faut, nous semble-t-il, désexualiser le viol. D'abord en admettant que des hommes et des femmes peuvent être les victimes de cet attentat et de fait le sont tous les jours; puis en reconnaissant que des femmes et des hommes sont les auteurs de cette agression, tous les jours. 
Pouvons-nous parler du viol comme nous en parlions il y a 20 ans?

Depuis plusieurs années, dans la majorité des pays occidentaux, de fort courants tendent à favoriser l'avortement sur demande; la complète liberté d'exercice de la sexualité dès l'adolescence avancée, avec tous les partenaires de ses choix successifs; la contraception et la vasectomie sur demande; le libre accès pour tous, hommes et femmes, à tous les lieux de leurs choix, aux heures de leurs choix; une égalité croissante des sexes dans les compétitions sportives, une grande liberté dans la mobilité physique et occupationnelle; le refus croissant des femmes d'être prises en charge et l'incapacité croissante de beaucoup d'hommes de prendre en charge pour toute une vie femme et enfants, etc. Il apparaît difficile de concilier ces progrès et cette libération avec les haut cris féminins contre les violeurs. La position qui consiste à réclamer l'emprisonnement et le châtiment exemplaire des violeurs n'est pas tenable. Il me semble que les féministes devraient être plus préoccupées de voir à l'instauration d'excellents cours d'éducation sexuelle qu'à la restauration des peines contre les violeurs. D'ailleurs, il serait plus logique d'apprendre aux femmes et aux hommes les conséquences de leurs nouvelles libertés et les moyens d'en tirer parti. Enfin, il ne serait peut-être pas superflu d'aider les hommes à entrer en rapport autrement que par la force brute ou l'évitement avec des femmes qui les affrontent, les contestent, les concurrencent ou les dépassent.

\section{BIBLIOGRAPHIE}

ALTHUSSER, L. (1976). «Idéologie et appareils idéologiques d'État», in Positions, Paris, Seuil.

ALLEN, F.A. (1964) 1974. $3^{\circ}$ édition The Borderland of Criminal Justice, Chicago, The University of Chicago Press.

ALLEN, F.A. (1981). The Decline of the Rehabilitative Ideal: Penal Policy and Social Purpose, New Haven, Yale University Press.

BECCARIA, C. (1764) 1965. Des délits et des peines, Genève, Dalloz.

BENTHAM, J. (1829-1830). Traités de législation civile et pénale, in OEuvres de J. Bentham, Bruxelles, Coster.

BERTRAND, M.A. (1977). «Le caractère inique et discriminatoire du système de justice des mineurs: les filles dites délinquantes au Canada», Déviance et Société, $1,2,187-202$.

BERTRAND, M.A. (1979). La femme et le crime, Montréal, L'Aurore. 
BERTRAND, M.A. (1980). «La non-intervention radicale», in Le contrôle social en état de crise, compte rendu de la $4^{\mathrm{e}}$ Conférence de criminologie appliquée, édité par Jacques Laplante, Ottawa, Université d'Ottawa, département de criminologie, p. 175-182.

COMMISSION DE RÉFORME DU DROIT DU CANADA (1975). Les confins $d u$ droit pénal, Ottawa, Information Canada.

COMMISSION DE RÉFORME DU DROIT DU CANADA (1976). Notre droit pénal, Ottawa, Information Canada.

DAVY, G. (1953). «Le droit, la justice», la morale, in l'Évolution humaine, tome IV, Paris, Quillet.

DEVLIN, P. (1965). The Enforcement of Morals, London, Oxford University Press.

FULLER, L.L. (1964). The Morality of Law, New Haven, Yale University Press.

HART, H.L.A. (1959). Law, Liberty and Morality, Stanford, Stanford University Press.

LANDREVILLE, P., V. BLANKEVOORT, A.P. PIRES (1980). Les coûts sociaux du système pénal, Rapport de recherche, Université de Montréal, École de criminologie.

MIAILLE, M. (1980). «Le droit-violence», Déviance ef société, 4, 2, 167-177 (réponse à Althusser).

MILL, J.S. (1859) 1970. On Liberty, Essential Works of J.S. Mill, New York, Bantam.

NIETZSCHE, F. (1885) 1971. Par-delà le bien et le mal, Paris, Gallimard/Idées.

NIETZSCHE, F. (1887) 1964. La généalogie de la morale, Paris, Gallinard/Idées.

PLATON (-797) 1978. The Laws, traduit et édité par T.J. Saunders, New York, Penguin.

POULANTZAS, N. (1978). L'état, le pouvoir et le socialisme, Paris, PUF.

SCHUR, E.M. (1973). Radical Non-Intervention, Englewood Cliffs, Prentice Hall.

STATISTIQUE CANADA $(1975,1976,1977,1978,1979,1980,1981)$. Statistique de la criminalité et de l'application des règlements de la circulation, Ottawa, ministère des Approvisionnements et Services.

TAYLOR, I. (1981). Law and Order : Arguments for Socialism, London and Basingstoke, The MacMillan Press Lid. 